\title{
Study on Optical Fiber Sensing for Detecting Liquid Penetration into Anti-corrosive Resins
}

\author{
Yui Hashimoto ${ }^{\mathrm{a}}$, Yusuke Hioka ${ }^{\mathrm{b}}$, Masatoshi Kubouchi ${ }^{\mathrm{a}, *}$

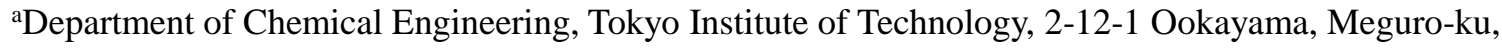 \\ Tokyo 152-8550, Japan \\ bDepartment of Mechanical Engineering, University of Auckland, Private Bag 92019, Auckland 1142, New Zealand \\ *Corresponding Author: mkubouch@chemeng.titech.ac.jp
}

\begin{abstract}
Anti-corrosive thermosetting resins are commonly used in chemical plants to prevent corrosion failure of chemical equipment. As they are often used in contact with corrosive liquid for a long time, liquid penetration into the resins causes problems. On-line sensing to detect the penetrating liquid into the resins is useful to prevent accidents caused by the liquid reaching to the crucial parts of the chemical equipment and to estimate the life-time of the equipment.

Penetration behavior of the liquid in an anti-corrosive resin can be described by Fick's second law, which includes the diffusion coefficient. The method proposed in this study estimates the diffusion coefficient using optical fiber sensor modules embedded in the different depths of the anticorrosive resin while the surface of the anti-corrosive resin is immersed in the liquid. The optical fiber sensor modules contain $\mathrm{pH}$ indicators on the light path of the fiber and the spectrum of the transmitted light is monitored. The diffusion coefficient is estimated from the difference between the time instants where the two sensor modules detect the liquid penetration.
\end{abstract}

Nevertheless the diffusion coefficient estimated by the proposed method in an experiment was about $40 \%$ smaller than that estimated by a conventional off-line measurement, the study reveals that the proposed method is able to estimate the diffusion coefficient by on-line manner. To further improve the estimation accuracy, the effect of the assumption set for the liquid concentration of the resin, which would have caused the error, is also discussed.

Keywords: optical fiber sensor, on-line corrosion monitoring, penetration, resin degradation, diffusion coefficient

\section{Introduction}

Anti-corrosive thermosetting resins are commonly used for coating the surface of chemical plants to protect metallic equipment from corrosion and as a matrix resin in fiberreinforced plastics (FRPs) for corrosive process fluid. As the surface of resins is often in contact with corrosive liquid, e.g. acid or alkali solution, for quite a long time, even anticorrosive resins would also degrade which may cause catastrophe such as explosion of the chemical plant. One of the causes of the degradation of anti-corrosive resins is the penetration of the liquid into resins. Liquid diffusion in the resins has significant influence on the properties and performance of anti-corrosive resins and $\operatorname{FRPs}^{(1,2)}$.

Sensing technologies to detect liquid penetration before it reaches to the critical parts of the equipment are important to prevent serious accidents caused by the degradation of resins. In addition, estimating intrinsic parameters of the resins such as the diffusion coefficient to describe the liquid penetration is required to predict the life-time of the equipment. Detecting liquid penetration in resins is important to estimate the corrosion behavior of resins. Two types of measurement technique to detect liquid penetration into resins are available; on-line measurement continuously checks a particular position inside the equipment in which a sensor is embedded, i.e. monitoring, whereas off-line measurement inspects equipment at some time interval. A variety of off-line measurements such as gravimetric liquid uptake evaluation ${ }^{(3)}$, attenuated total reflection Fourier transform infrared spectroscopy (ATR-FTIR) analysis ${ }^{(4)}$, and penetration depth evaluation with Energy Dispersive X-ray Spectroscopy $(\mathrm{EDS})^{(5)}$ have been used to estimate the diffusion coefficients in resins. However these off-line 
measurement methods are destructive to resins hence they are inapplicable to field equipment ${ }^{(6)}$. In addition, because resins in chemical plants are used in various conditions, it is difficult to reproduce the same condition in experiments. Thus an on-line method to estimate the penetration rate of liquid in resins for actual equipment is highly required.

In the previous studies of the authors, an optical fiber sensor with $\mathrm{pH}$ indicator was developed and embedded in a resin to detect penetrating solution, which was then installed in chemical storage tank modules ${ }^{(7,8)}$. This paper proposes a sensing method to estimate the diffusion coefficient of Fick's second law $^{(9)}$ to describe the liquid penetration in an anticorrosive thermosetting resin using an on-line measurement with two optical fiber sensors. Since the proposed measurement is a non-destructive method, it is a practicable technique that provides effective information for predicting the degradation of the resins.

The diffusion coefficient was calculated using the difference of the detecting times at plural sensors embedded in different depths from the surface of the resin. The result measured by the proposed method was compared to the diffusion coefficient calculated by an off-line measurement.

\section{Proposed Method}

The proposed method utilizes two sensors, i.e. sensor A and $\mathrm{B}$ embedded in an anti-corrosive resin in different depths from the surface, $l_{\mathrm{a}}$ for sensor A, and $l_{\mathrm{b}}$ for sensor B $\left(l_{\mathrm{a}}<l_{\mathrm{b}}\right)$, to estimate the diffusion coefficient. When the liquid penetration into the resin is described by Fick's second law given by Eq. (1),

$$
\frac{\partial C}{\partial t}=D \frac{\partial^{2} C}{\partial x^{2}}
$$

the concentration distribution in the resin can be calculated by explicit methods ${ }^{(9)}$ :

$$
\frac{C_{t+1, x}-C_{t, x}}{\Delta \tau}=D \frac{C_{t, x+1}+C_{t, x-1}-2 C_{t, x}}{\Delta x^{2}}
$$

where $C_{t, x}$ is the normalized concentration of the liquid in the resin at time $t$ at position $x$ with respect to the molar concentration at the surface of the resin $C_{t, 0}^{\prime}$, i.e. $C_{t, x}=$ $C_{t, x}^{\prime} / C_{t, 0}^{\prime}$. Note that $C_{t, x}^{\prime}$ denotes the molar concentration of the liquid in the resin at time $t$ at position $x$ in contrast to the normalized concentration. Here $C_{t, 0}^{\prime}$ is assumed to be equal to the molar concentration of the solution in contact with the resin.

$D$ is the diffusion coefficient which is assumed to be a constant value at a constant temperature. In Eq. (2), $C_{0, x}=0$ $(x>0), C_{t, 0}=1(t>0)$ are used for the boundary conditions

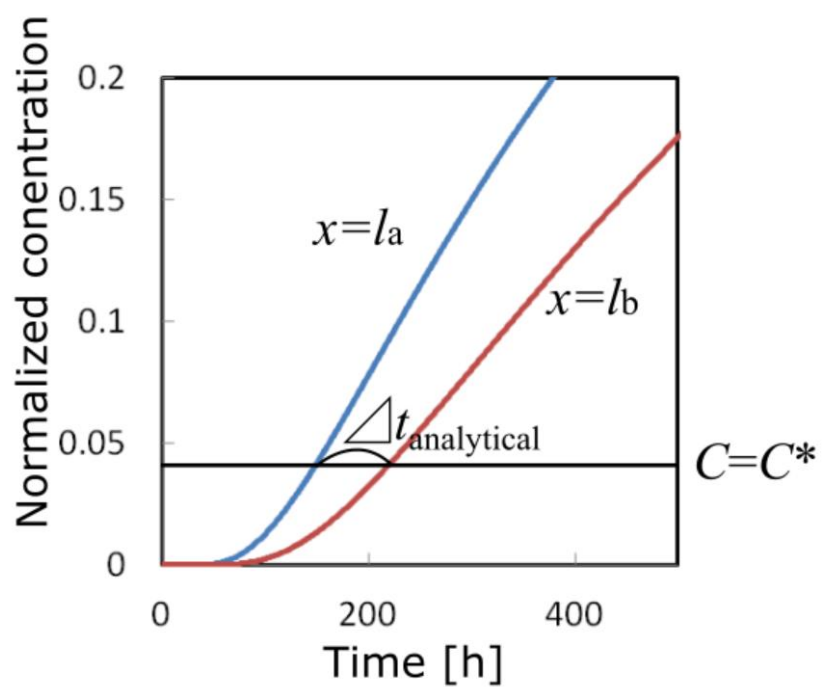

Fig. 1. Normalized concentration at different positions in a resin calculated by Eq. (2). The detecting time difference is defined by the time difference between the instants where the normalized concentration reaches to $C^{*}$ at the two sensor positions (Eq. (4)).

to describe the penetration from one side of the resin. For each diffusion coefficient, the time series of the normalized concentrations at positions $x=l_{\mathrm{a}}$ and $x=l_{\mathrm{b}}$ are calculated by Eq. (2) and are drawn as shown in Fig. 1.

Given the spectrum of the transmitted light through the $\mathrm{pH}$ indicator of the optical fiber sensors changes at the hydrogen concentration $\left[\mathrm{H}^{+}\right]^{*}$, the relationship between $\left[\mathrm{H}^{+}\right]^{*}$ and the normalized concentration of the liquid at the position of sensor, $C^{*}$ is calculated from Eq. (3), where $\alpha$ is the degree of the ionization.

$$
\left[\mathrm{H}^{+}\right]^{*}=\alpha C_{t, x}^{\prime}=\alpha C^{*} \cdot C_{t, 0}^{\prime}
$$

The difference between the time instants when the penetration is detected by sensors A and B is defined by Eq. (4) as the analytical detecting time difference.

$$
\begin{gathered}
\Delta t_{\text {analytical }}(D)=t\left(C_{t, l_{\mathrm{b}}} \mid C_{t, l_{\mathrm{b}}}=C^{*}\right) \\
-t\left(C_{t, l_{\mathrm{a}}} \mid C_{t, l_{\mathrm{a}}}=C^{*}\right)
\end{gathered}
$$

Calculating $\Delta t_{\text {analytical }}$ for different diffusion coefficients provides a relationship between $D$ and $\Delta t_{\text {analytical for a }}$ specific $C^{*}$ (this will be further discussed in Section 4.3). Thus the diffusion coefficient can be determined by searching $D$ which provides $\Delta t_{\text {analytical }}(D)$ closest to the actual detecting time difference $\Delta t_{\text {actual }}$ defined by:

$$
\Delta t_{\text {actual }}=\left|t_{\mathrm{b}}-t_{\mathrm{a}}\right|
$$

where $t_{\mathrm{a}}$ is the detecting time of penetration by sensor $\mathrm{A}$ and $t_{\mathrm{b}}$ is that by sensor $\mathrm{B}$. 


\section{Experiment}

\subsection{Materials}

Isophthalic acid unsaturated polyester is an anti-corrosive thermosetting resin and is commonly used as a matrix resin of FRPs. In this study, RIGORAK® (Showa Denko Co, Japan) and methyl ethyl ketone peroxide PERMEK® (Nof Corporation, Japan) were used to prepare the resin. Vinyl ester resin RIPOXY R-804® (Showa Denko Co, Japan) was used as a support part.

Plastic optical fiber ESKA® (Mitsubishi Rayon Co., Japan) was used in a sensor module to detect the liquid penetration. Bromophenol blue was used as a $\mathrm{pH}$ indicator. Epoxy resin EPOMIK® R-140 (Mitsui Chemicals, Inc., Japan) and ammine hardener JEFFAMINE® D-230 (Hantsman Co.) were mixed and then placed on the light path of the fiber. Glass mats and glass crosses were used to support the axes of the fibers.

\subsection{Preparation}

\subsubsection{The sensor module}

The optical axes of the plastic optical fibers were supported by isophthalic acid unsaturated polyester resin, glass mats, and glass crosses. Slits with the width of $1 \mathrm{~mm}$ were cut on the fibers, and mixtures of the epoxy resin, ammine hardener, and bromophenol blue ethanol solution $(0.1 \mathrm{~g} / 10 \mathrm{~mL}$-ethanol $)$ in the weight ratio of $100: 33: 4$ were poured into the slits. By curing them in an oven at $30{ }^{\circ} \mathrm{C}$ for 24 hours, the sensor module was prepared (Fig. 2).

\subsubsection{Preparation of the sample}

Vinyl ester resin was cured in a cylindrical shape with 80 $\mathrm{mm}$ in diameter and $20 \mathrm{~mm}$ in height. A bottomed hole with $32 \mathrm{~mm}$ in diameter and the two tunnels in different depths where fiber sensor modules pass through were machined on the disk shaped resin. By pouring non-cured unsaturated polyester resin into the case, the sensor modules were embedded in the resin (Fig. 3). The samples were cured at 50 ${ }^{\circ} \mathrm{C}$ for 24 hours, and the surface was smoothed by a lathe machining.

\subsection{Immersion test}

One side of the resin was immersed in the 20 mass $\%$ (6.00 mol/L) hydrogen-chloride aqueous solution at $50{ }^{\circ} \mathrm{C}$ (Fig. 4). The spectrum of the transmitted light through the optical fiber was recorded through the experiment using light source and Spectrometers (USB2000; Ocean optics
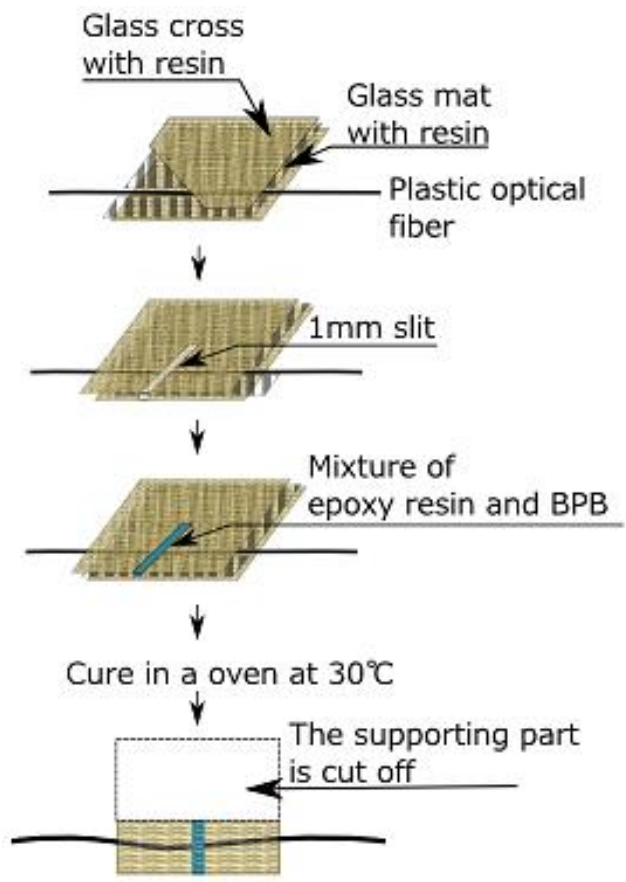

Fig. 2. Preparation of the sensor modules.

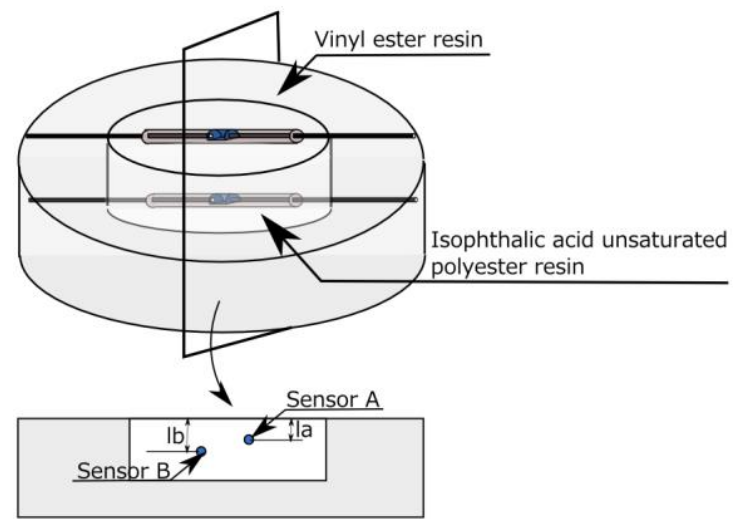

(a) Schematic view of the sample and its cross-section image

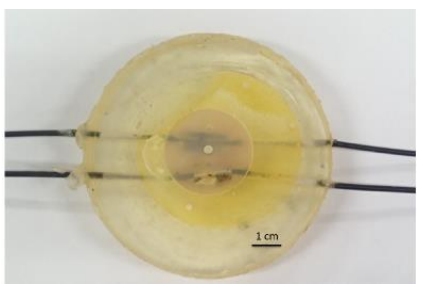

(b) Photo of the sample after 500 hours immersion test

Fig. 3. Sample with two embedded sensor modules.

Inc.). Scanning Electron Microscope (SEM) and Energy Dispersive X-ray Spectroscopy (EDS) (JSM-6510LA; $J E O L$ ) were used for the analysis of the cross section of the resin after 500 hours immersion test. The embedded depths of sensor A and B were measured by the results of SEM analysis. 


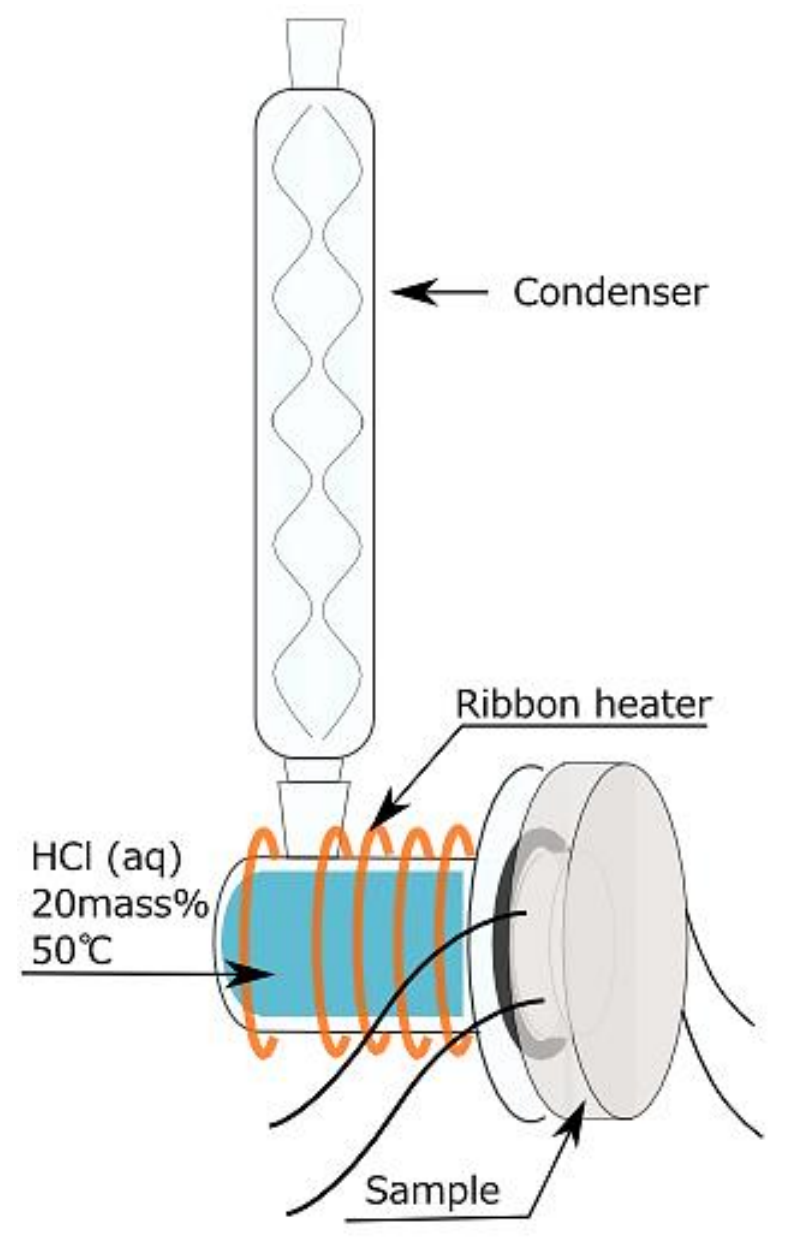

Fig. 4. Experimental setup of one side immersion test.

\section{Results and Discussion}

\subsection{Transmittance monitoring}

The absorbance of the transmitted light through the optical fiber was monitored by the spectrometer. The absorbance at the wavelength $640 \mathrm{~nm}$, the color of orange, decreases when the hydrochloride aqueous solution reached to the sensor after penetration in the unsaturated polyester resin (Fig. 5). Mixture of the epoxy resin and bromophenol blue changed its color from blue to yellow as $\mathrm{pH}$ in the indicator resin decreased when acid solution reached to the sensor. Given the spectrum change is detected between the time instances $t_{i 1}$ and $t_{i 2}$, the detecting time $t_{i}$ is calculated by:

$$
t_{i}=\frac{t_{i 1}+t_{i 2}}{2}
$$

where $i$ takes either a or b. Thus from Fig. 5 the detecting time of sensor $\mathrm{A}$ is $t_{\mathrm{a}}=177$ hours and that of sensor $\mathrm{B}$ is $t_{\mathrm{b}}=367$ hours, which provide the measured actual detecting time $\Delta t_{\text {actual }}=\left|t_{\mathrm{b}}-t_{\mathrm{a}}\right|=190$ hours.

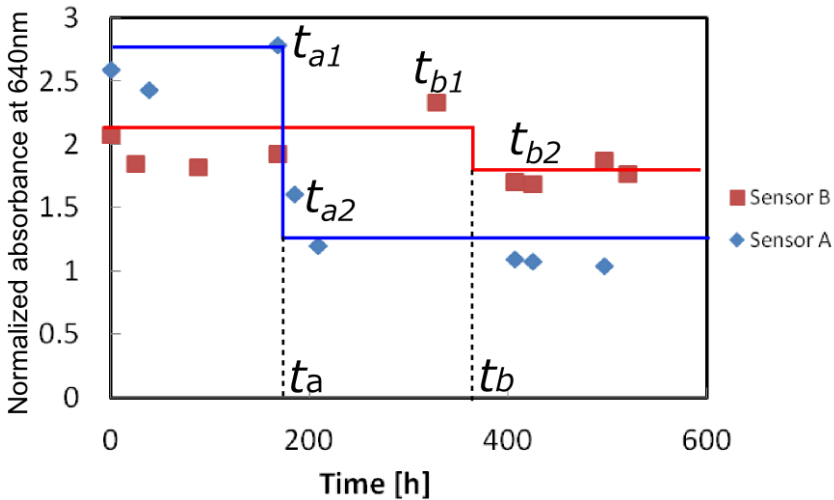

Fig. 5. Normalized absorbance at $640 \mathrm{~nm}$ of the transmitted light based on the absorbance at $800 \mathrm{~nm}$ through optical fiber sensor modules.

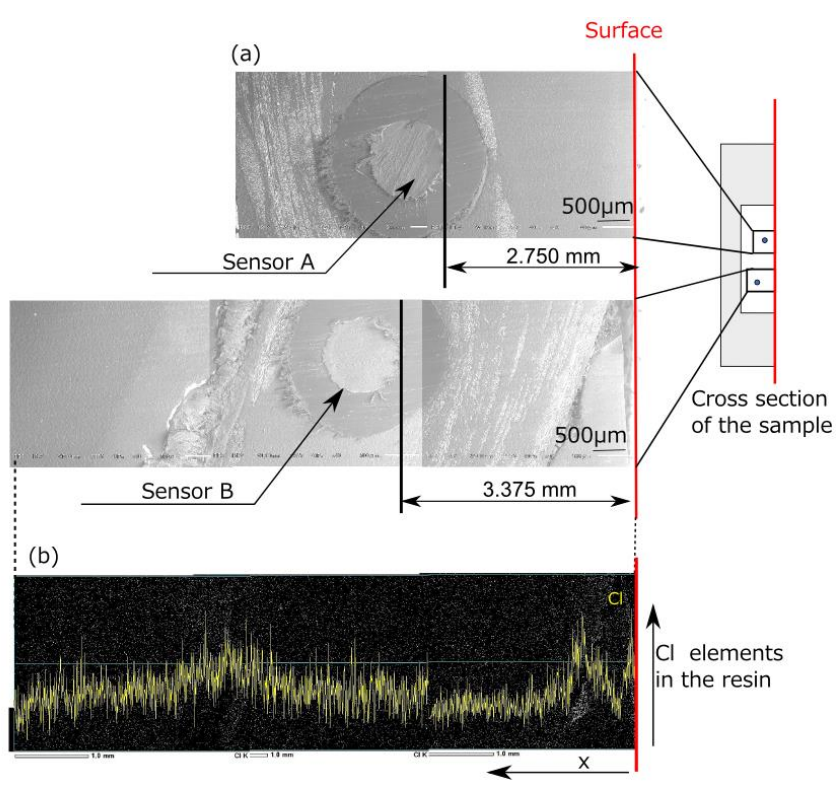

Fig. 6. Cross-section analysis of the sensor modules embedded in the resin after immersion in hydrogen chloride aqueous solution for 500 hours: (a) SEM observation in the proximity of the sensors, (b) EDS analysis of the chloride element in the observed area of sensor B.

\subsection{Cross section analysis}

The resin after immersion in hydrogen chloride aqueous solution for 500 hours was cut into small pieces and the cross sections were examined by SEM and EDS as shown in Fig. 6. The sensors were embedded in the isophthalic acid unsaturated polyester resin in the depth of $l_{\mathrm{a}}=2.750 \mathrm{~mm}$ for sensor A and $l_{\mathrm{b}}=3.375 \mathrm{~mm}$ for sensor $\mathrm{B}$, respectively. EDS analysis showed that chloride iron from hydrochloride aqueous solution had reached to a deeper depth than that of sensor B after 500 hours immersion test. This result agrees with the result shown in Fig.5. 


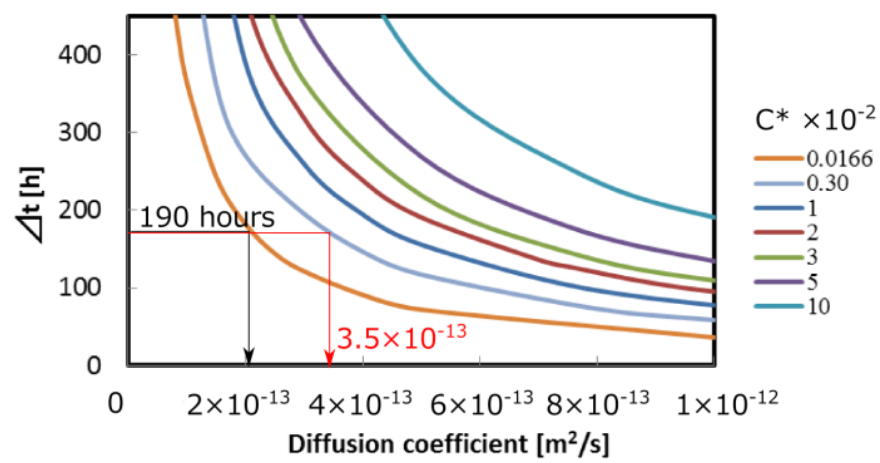

Fig. 7. Estimating diffusion coefficient by the detecting time difference of the embedded sensors whose depths are $l_{\mathrm{a}}=2.750 \mathrm{~mm}$ for sensor $\mathrm{A}$ and $l_{\mathrm{b}}=3.375 \mathrm{~mm}$ for sensor B.

\subsection{Diffusion coefficient estimation}

Bromophenol blue changes its color from blue to yellow at $\mathrm{pH}=3^{(10)}$, which means that $=10^{-3}$ was used in the experiment. Thus $C^{*}=1.66 \times 10^{-4}$ provided $C_{t, 0}^{\prime}=6.00$ $\mathrm{mol} / \mathrm{L}$ and $\alpha=1$ for hydrogen chloride aqueous solution. Since the measured actual detecting time difference $\Delta t_{\text {actual }}=190$ hours the estimated diffusion coefficient of hydrogen chloride aqueous solution in isophthalic acid unsaturated resin was $2.00 \times 10^{-13} \mathrm{~m}^{2} / \mathrm{s}$ (Fig. 7). This result agrees with the order of $D$ value obtained by the off-line measurement stated later.

\subsection{Off-line measurement of diffusion coefficient}

For evaluating the adequacy of the diffusion coefficient obtained by the proposed method, the diffusion coefficient was also estimated by an off-line measurement. In the offline measurement, the diffusion coefficient was estimated by the relationship between the penetration depth of the liquid in the resin and the time elapsed in an immersion test.

The immersion test was conducted by dipping plate shaped small pieces of isophthalic acid unsaturated polyester resin in the hydrogen chloride aqueous solution (20 mass\%) at $50{ }^{\circ} \mathrm{C}$ for many hours. To measure the penetration depths, the cross-sections of the resins were analyzed by SEM and EDS after the resins were immersed in the liquid for time $t$. Because it was difficult to determine the deepest points to which the chloride element reached, the penetration depth $x$ was characterized by the length twice as long as the half width half maximum (HWHM) by assuming that the distribution of the chloride element penetrating into the resin was unimodal whose peak was located at the surface of the resin, i.e. $x=0$, as shown in Fig. 8(b). (a)

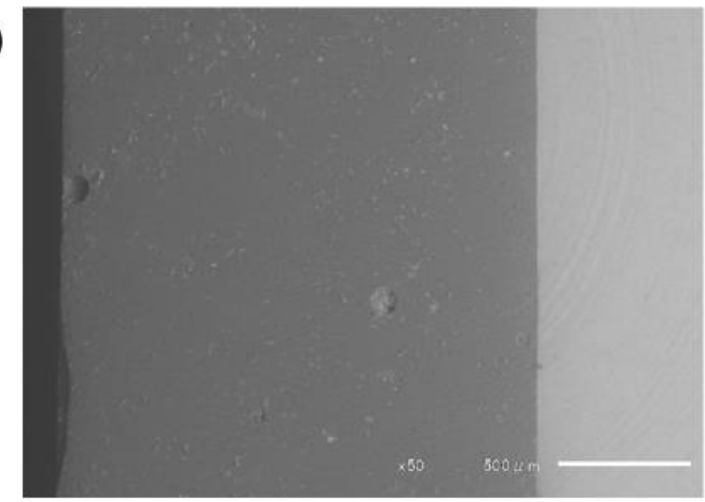

(b)

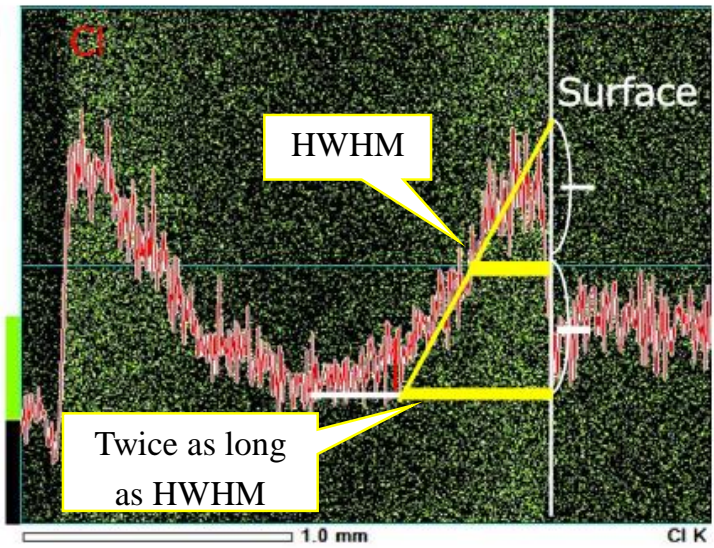

Fig. 8. Cross section analysis of the isophthalic acid unsaturated resin after immersed in the hydrogen chloride aqueous solution for 73 hours: (a) SEM observation, (b) EDS analysis and definition of the penetration depth.

The relationship between the mass of the diffusing liquid which has penetrated into a resin at the time $t$ and after the penetration has saturated, i.e. $M_{t}$, and $M_{\infty}$, respectively, is expressed by Eq. $(7)^{(9)}$. In the initial stage of the diffusion, Eq. (7) can be approximated by Eq. (8) because the second term in the bracket is much smaller than the first term.

$$
\frac{M_{t}}{M_{\infty}}=2\left(\frac{D t}{l^{2}}\right)^{\frac{1}{2}}\left\{\pi^{-1 / 2}+2 \sum_{n=1}^{\infty}(-1)^{n} \operatorname{ierfc} \frac{n l}{\sqrt{D t}}\right\}
$$

Here $l$ and ierfc are the thickness of the resin and the integral of the complementary error function, respectively.

$$
\frac{M_{t}}{M_{\infty}}=2\left(\frac{D t}{\pi l^{2}}\right)^{\frac{1}{2}}
$$

The left side of Eq. (8) can be approximated by the ratio of the volume of the liquid penetrating into the resin at $t$ and that after the penetration has saturated if the density of the liquid is assumed to be constant. Then the ratio of the volume can be approximated as $x / 2 l$ by assuming that the distribution of the liquid penetrating into the resin has a triangle 


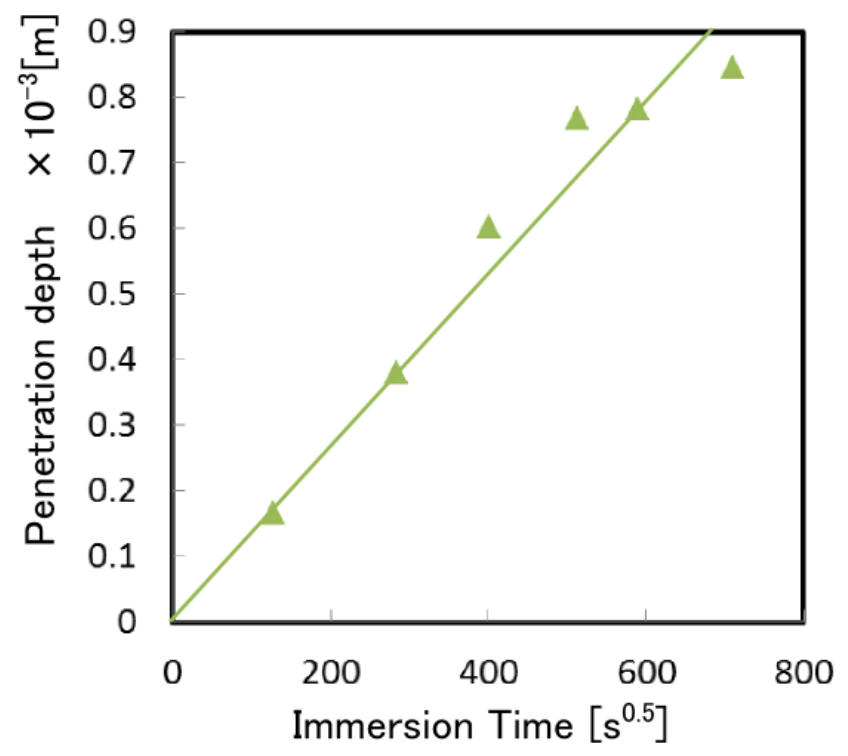

Fig. 9. Relationship between the penetration depth and immersion time.

prism with a constant immersed area. Thus the Eq. (8) can be calculated into Eq. (9).

$$
x=4 \sqrt{\frac{D t}{\pi}}
$$

Hence the diffusion coefficient $D$ can be calculated from the slope of the penetration depth with respect to immersion time $t$.

\subsection{Discussion}

Fig. 9 shows the penetration depths $x$ measured at various time $t$ in the immersion test. The slope of the relationship between $x$ and $t$ was calculated as $1.33 \times 10^{-6} \mathrm{~m} / \mathrm{s}^{1 / 2}$ by linear regression using the least squares method. This slope was equal to $4 \sqrt{D / \pi}$ in the right side of Eq. (9). Thus the diffusion coefficient was calculated as $3.47 \times 10^{-13} \mathrm{~m}^{2} / \mathrm{s}$.

The diffusion coefficients obtained by the proposed method and the off-line measurement are summarized in Table 1. The diffusion coefficient estimated by the proposed on-line method was $42.4 \%$ smaller than that estimated by the off-line measurement.

This error would have been caused by the erroneous value being set to the normalized concentration at the position of the sensor, i.e. $C^{*}$. From Fig. $7, C^{*}$ which would give a diffusion coefficient similar to that estimated by the off-line measurement, i.e. $D=3.47 \times 10^{-13}$, at the time difference $\Delta t_{\text {actual }}=190$ hours was $C^{*}=3 \times 10^{-3}$.
Table 1. Estimated diffusion coefficient at $50{ }^{\circ} \mathrm{C}\left[\mathrm{m}^{2} / \mathrm{s}\right]$.

\begin{tabular}{|c|c|c|}
\hline On-line measurement & Off-line measurement & Error [\%] \\
\hline $2.00 \times 10^{-13}$ & $3.47 \times 10^{-13}$ & 42.4 \\
\hline
\end{tabular}

Considering the relationship in Eq. (3), where $\left[\mathrm{H}^{+}\right]^{*}$ should be a fixed value, the molar concentration of the liquid in the resin at the surface, i.e. $C_{t, 0}^{\prime}$ should have been much smaller than that of the hydrogen chloride aqueous solution. If $C^{*}=3 \times 10^{-3}, C_{t, 0}^{\prime}$ should be $0.33 \mathrm{~mol} / \mathrm{L}$ to provide $\left[\mathrm{H}^{+}\right]^{*}=10^{-3}$ where the $\mathrm{pH}$ indicator changes its color. This means that $C^{\prime}{ }_{t, 0}$ should have been only $5.50 \%$ of the molar concentration of the solution. The assumption that the molar concentration at the surface of the resin was equal to that of the solution in contact with the resin would not hold. In fact, another experiment conducted by the authors showed that the mass uptake of the isophthalic acid unsaturated polyester resin could be at most $2 \%$ of the weight of the resin when they were immersed in the hydrogen chloride aqueous solutions $\left(20\right.$ mass $\%$ ) at $50{ }^{\circ} \mathrm{C}$. This agreed with the finding that $C_{t, 0}^{\prime}$ should have been much smaller than the molar concentration of the hydrogen chloride aqueous solution.

Further investigation of the relationship between the concentrations at the surface of the resin and the solution in contact with the resin are required in order to improve the estimation accuracy of the proposed method.

\section{Conclusions}

The diffusion coefficient of Fick's second law in isophthalic acid unsaturated resins for describing the liquid penetration behavior was estimated by two sensor modules embedded in different depths from the surface of the resin. The diffusion coefficient estimated by the proposed method was compared to the diffusion coefficient estimated by the off-line measurement. The estimated values were slightly different because the normalized concentration $C^{*}$ had not been properly given due to the assumptions set for the molar concentration of the surface of the resin. However the proposed method will allow for providing useful information about resin degradation by a non-destructive and easily applicable way.

Further investigations on the concentration of the liquid in the resin will be required to estimate the diffusion coefficient with better accuracy. 


\section{References}

(1) Abastari, Tetsuya Sakai, Hideki Sembokuya, Masatoshi Kubouchi, and Ken Tsuda : "Study on permeation behavior and chemical degradation of PA66 in acid solution", Polymer Degradation and Stability, Vol. 92, No. 3, pp. 379-388, 2007

(2) A. Bledzki, R. Spaude, and G. W. Ehrenstein : "Corrosion Phenomena in Glass Fibers and Glass Fiber Reinforced Thermosetting Resins", Composites Science and Technology, Vol. 23, No. 4, pp. 263-285, 1985

(3) Antoine Guiu, and Martin E.R. Shanahan : "Effects of solvent exposure on the adhesion of grafted PE to EVOH copolymer", International Journal of Adhesion and Adhesives, Vol. 24, No. 6, pp. 523-531, 2004

(4) Małgorzata Krzak, Zbisław Tabor, Paweł Nowak, Piotr Warszyński, Anastasis Karatzasc, Ioannis A. Kartsonakis, and George C. Kordas : "Water diffusion in resin coatings containing water-trapping particles. Part 2. Experimental verification of the mathematical model”, Progress in Organic Coating, Vol. 75, No. 3, pp. 207-214, 2012

(5) Maryam Mohri, Mahmoud Nili-Ahmadabadi, and Stefan Flege : "Diffusion evaluation of $\mathrm{Cu}$ in NiTi Bilayer thin film interface", Journal of Alloys and Compounds, Vol. 594, No. 5, pp. 87-92, 2014

(6) Yui Hashimoto, Saiko Aoki, Masatoshi Kubouchi, and Tomonori Tomiyama : "Sensor for Detecting Alcohol Penetration into Anti-corrosion Resin”, Proc. 8th AOTULE Postgraduate Conference and Workshop 2013, p. 43, 2013

(7) Tomohiro Gotou, Masashi Noda, Tomonori Tomiyama, Hideki Sembokuya, Masatoshi Kubouchi, and Ken Tsuda : "In situ health monitoring of corrosion resistant resins exposed to alkaline solutions using $\mathrm{pH}$ indicators", Sensors and Actuators B: Chemical, Vol. 119, No. 1, pp. 27-32, 2006

(8) Yui Hashimoto, Saiko Aoki, Nobuo Mitomo, and Masatoshi Kubouchi : "Application study on resin degradation sensor for FRP storage tank", Industrial, Chemical Machinery \& Safety Division Conference 2013 fall, pp. 9-10, 2013

(9) John Crank: "The mathematics of diffusion. Second Edition”, Oxford, UK, Clarendon Press, pp. 137-159 1975

(10) Jacqueline Ferreira, and Emerson M. Girotto : "Optical pH sensitive material based on bromophenol blue-doped polypyrrole", Sensors and Actuators B: Chemical, pp.426-431, 2009 